\title{
Nuevas formas organizacionales en la gobernabilidad transnacional
}

\author{
New organizational forms in the transnational governance
}

José Gpe. Vargas Hernández ${ }^{1}$

\section{Resumen}

El objetivo de este trabajo es analizar las transformaciones que están teniendo las formas organizacionales para adaptarse a los requerimientos de una gobernabilidad transnacional que de respuesta a los cambios del medio ambiente creado por los procesos de globalización económica. A partir de un análisis de los elementos sobresalientes de las estructuras en las organizaciones, se determinan las tendencias en las nuevas formas organizacionales considerando las variables de un medio ambiente caracterizado por altos niveles de absorción de incertidumbre, complejidad de sistemas involucrados, flexibilidad de estructuras, comportamientos y procesos, interdependencia e interacciones de agentes económicos y actores políticos y sociales, incremento de la velocidad, cambios de poder y desmembramiento de fenómenos organizacionales. Finalmente, se hace una elaboración de las implicaciones del diseño organizacional e institucional bajo los diferentes modelos desde los enfoques del neoliberalismo, el intergubernamentalismo y las redes de gestión. A partir de este análisis se concluye con una delimitación de los principales retos.

Palabras clave: Formas organizacionales, gobernabilidad transnacional, nuevas formas organizacionales.

\begin{abstract}
The aim of this paper is to analyze the transformations that are leading the organizational forms to adapt to the requirements of transnational governance which is the response to environmental changes created by the economic globalization processes. Beginning from the analysis of outstanding features of organizational forms, the new organizational forms' tendencies are determined taking into consideration the environmental variables characterized by high levels of uncertainty absorption, complexity of involved systems, structure, behavior and processes flexibility, interdependence and interaction of economic agents and political and social actors, increments of speed, changes of power and break up of organizational phenomena. Finally, an elaboration of the organizational and institutional design implications under the different models since the neoliberal, intergovernmentalism and management networking approaches was made. Based on this analysis, the research is concluded with a delimitation of the main challenges.
\end{abstract}

Key Words: Organizational forms, transnational governance, new organizational forms.

\section{Formas organizacionales}

Todas las formas de organización despliegan un conjunto de elementos característicos. Las formas organizacionales se caracterizan por la identificación y diseminación de las metas colectivas, la regulación de los flujos de recursos organizacionales y la identificación y gobierno de derechos y obligaciones, funciones y papeles de los miembros de la organización.

Las estructuras y formas organizacionales son irracionales o no racionales en su carácter. Las organizaciones afectan las reglas y las políticas mediante procesos de percepción, interpretación y negociación. Todas las

M.B.A.; Ph.D.Economics and Public Administration. Profesor Investigador Instituto tecnológico de Ciudad Guzmán. Endereço: Cerrada Petronilo López 31 Centro - Ciudad Guzmán - Jalisco/México - CEP: 49000. E-mail: jvargas2006@gmail.com.

Artigo submetido em janeiro e aceito em abril de 2008 
posibles formas de organización constituyen modos alternativos de gobernabilidad con diferentes fortalezas y debilidades. En particular, los mercados, empresas y gobiernos como modos de gobernabilidad tienen ventajas relativas. Según Williamson (1991a,b), la empresa constituye la forma de organización que puede ser usada cuando todas las demás formas de organización fallan, estas satisfacen más eficientemente las demandas del mercado, incluso mejor que los mercados mismos (Coase, 1991). No obstante, hay acuerdo en que todas las posibles formas de organización tienen diferentes modos de gobernabilidad que intrínsecamente cuentan con debilidades y fortalezas, además de que presentan un conjunto de elementos característicos de cada una de las formas.

Las organizaciones y sus participantes ejercen influencia en las estructuras de gobernanza. Los nuevos sistemas de gobernanza introducen la desregulación en áreas donde existe una fuerte regulación, bajo diferentes estructuras, creando y legitimando las formas organizacionales. La creación y transformación de las instituciones requieren de la condición de procesos de legitimación para hacerlas más atractivas. Estos procesos pueden tener lugar en períodos cortos y largos, de forma abrupta, en episodios o en forma incremental.

La diferenciación de estructuras de gobernanza contribuye a la funcionalidad de las instituciones y organizaciones. Las instituciones, de acuerdo a North (1984) se definen como: (i) un conjunto de limitaciones a la conducta en la forma de reglas y regulaciones; (ii) como un conjunto de procedimientos para detectar desviaciones de las reglas y de las regulaciones; (iii) como un conjunto de normas de conducta éticas y morales, las cuales definen los contornos que constriñen las formas en las cuales las reglas y las regulaciones son especificadas y aplicadas. Las reglas no son simples sistemas regulatorios sino marcos de referencia cognitivos culturales que definen la naturaleza de los actores, sus intereses y sus derechos. La institucionalización de estándares normas y prácticas dentro de los campos organizacionales tienden a perpetuarse a través de procesos deliberados y políticos. Los procesos normativos y cognitivos puestos en movimiento por iniciativas legislativas y políticas establecen mecanismos coercitivos.

La lógica institucional determina la importancia de los problemas. Cuando la lógica institucional cambia, presiona a las formas organizacionales existentes a modificarse en sus estructuras, las que a su vez afectan la gobernanza. Selznick $(1949,1957)$ afirma que los valores institucionales y sus prácticas son precarios y sujetos a desplazamiento cuando son mal definidos, confusos o discrepantes. De esta forma, los requerimientos técnicos están en conflicto con su mantenimiento, las organizaciones tienen crisis financieras o demandas por eficiencia, en dependencia de constituyentes que no los apoyan, las organizaciones carecen de legitimidad y reconocimiento, disentimientos en la política interna, las elites que los protegen carecen de autonomía, poder o compromiso.

Algunos de los principios en que se sustenta la lógica institucional de las nuevas formas organizacionales, expresan que la teoría del hombre económico que busca maximizar sus beneficios (rational choice) se concreta en los incentivos económicos que proveen las instituciones, es decir, el individuo realiza relaciones oportunistas de intercambio en formas de organización que dan origen a mercados, jerarquías, etc.

Las transacciones económicas en el capitalismo ocurren en un mercado, siempre en sinergia, entre las empresas e individuos o en las jerarquías al interior de las grandes corporaciones y los Estados imperialistas. Para mundializar las relaciones económicas, las corporaciones transnacionales transforman las formas sociales, económicas, productivas y tecnológicas mediante procesos de relocación macroregional y regional. En el período expansivo de las corporaciones transnacionales, estas evaden los controles de cambios monetarios y regulación económica y financiera establecida por las instituciones de Bretton Woods, para transitar a la creación de instituciones de regulación transnacional. Las macroregiones se forman con la integración de varios países en bloques económicos.

Las fronteras organizacionales son formadas por arreglos regulativos y de gobernanza, concepciones cognitivas y culturales. Las fronteras de las organizaciones no son estáticas sino que cambian eficientemente de forma que puedan adaptarse a la evolución de las tecnologías. Coase (1937) explicó que las fronteras organizacionales dependen más de los costos de transacción que de la tecnología productiva. 
La propuesta de la economía de los costos de transacción a la teoría de las organizaciones es la del principio de adaptación, la cual puede ser de dos tipos: adaptación autónoma y adaptación cooperativa (Williamson, 2000). La adaptación autónoma de las organizaciones responde a los cambios en los precios relativos y la adaptación cooperativa se logra a través de la administración. La adaptación involucra costos de transacción más bajos y más altos resultados del cambio que la reforma. La economía de los costos de transacción proporciona las base para la economía mediante el diseño de estructuras de las nuevas formas de organización y por lo tanto, las consecuentes formas de gobernabilidad.

Las organizaciones políticas estatales actúan dentro de la frontera del Estado, tales como los tres niveles de gobierno y los poderes judicial y legislativo, mientras que las organizaciones políticas no estatales son las organizaciones voluntarias que surgen en la misma sociedad. Las instituciones políticas impactan lo económico e influyen en las decisiones. El diseño de instituciones político-jurídicas que garanticen el intercambio mediante la libertad contractual y los derechos de la propiedad, es una condición que opere el libre mercado bajo ciertas limitaciones en un sistema democrático.

La sociedad política legaliza las acciones de la sociedad civil y ordena su funcionamiento con una orientación hacia la esfera pública. Es la sociedad civil la que instituye la función pública mediante la creación de un arreglo constitucional, aunque a largo plazo, no está exenta de riesgos, la sociedad civil tiene la tendencia a consolidar la democracia, más que a fundarla, aunque este es un planteamiento cuestionable. La esfera pública no-estatal es el espacio en que comparten el poder el Estado y la sociedad, más centrada en las organizaciones sociales que promueven el talento innovador, emprendedor y gerencial bajo formas organizativas de cogestión social. La aceptación social de los emprendedores puede ser baja en sociedades altamente igualitarias en un Estado de Bienestar debido a las actitudes y a decisiones políticas que son inducidas por estas actitudes.

La reestructuración de las formas de organización social que permitan crear nuevos mecanismos de colaboración y solidaridad, facilitan la formación del tejido social que fortalecen y dotan las normas, instituciones y organizaciones que facilitan el funcionamiento del mercado. Las redes con orientación hacia la toma de decisiones fortalecen la democracia en tanto que permite al mecanismo de toma de decisiones en los niveles más bajos de la organización social sin que sean confinados por la burocracia a instituciones formales. La idea original de la democracia, a diferencia del republicanismo, mezcla las esferas privada y pública que se proyectan en el establecimiento de mecanismos institucionales y organizacionales con una tendencia efectiva del poder público y con una mayor tendencia hacia el control, como en el caso de las democracias consociativas o consensuales. Las decisiones democráticas son públicas mediante procedimientos especificados en normas legales públicas. Los miembros de una organización tienen como expectativas solucionar problemas de acción colectiva para proveerse de algunos bienes.

La gestión privada funciona en organizaciones específicas a nivel micro. La prevalencia de las diferentes formas organizacionales entre los países más desarrollados y los menos desarrollados depende de los costos diferenciales de materias primas y mano de obra, entre otros factores. La reducción en los costos relativos de la subcontratación extranjera eleva la prevalencia de formas organizacionales con base en importación de bienes lo cual afecta su atractividad con respecto a la integración. Cuando un productor final de bienes es dueño de la unidad de producción de componentes localizada en un país desarrollado, la forma organizacional es una integración vertical, mientras que la unidad de producción de insumos intermedios localizada en los países menos desarrollados la forma organizacional es una integración con una vertical inversión extranjera directa que genera comercio internacional intrafirma.

La integración vertical está cambiando rápidamente en los ambientes tecnológicos y ofrece ventajas de desempeño sobre las estructuras horizontales. En una investigación de Antrás y Helpman (2003), los investigadores encontraron que las empresas de alta productividad adquieren bienes intermedios en el Sur, mientras que las empresas de baja productividad en el Norte. Las empresas que adquieren insumos en el mismo país, que están dentro del grupo de baja productividad subcontratan, mientras que, las de alta productividad realizan integraciones. 


\section{Nuevas formas organizacionales}

En la complejidad e incertidumbre de los entornos actuales, el sistema de gobernabilidad debe ser lo suficientemente flexible para dar oportunidad a que se expresen las expectativas y se resuelvan los conflictos de intereses entre los diversos actores estratégicos. La flexibilidad de un sistema organizacional - institucional para aplicar y adaptar las reglas y procedimientos es el principal sustento para garantizar la gobernabilidad en ambientes turbulentos, dinámicos, de alta complejidad e incertidumbre.

Las nuevas formas organizacionales son requerimientos a los cambios en las metas de las organizaciones para responder a la incertidumbre, la complejidad, el enfoque estratégico en el diseño de procesos y estructuras, un énfasis en lo social e interpersonal y una reemergencia de la legitimidad. Más que los puntos de vista de las organizaciones como estructuras estables diseñadas para absorber la incertidumbre, las nuevas organizaciones se construyen en la flexibilidad y la capacidad para manejar cambio constante, por lo que es importante lograr la alineación de los componentes organizacionales para preservar la flexibilidad y construir sistemas que se enfrenten con esta paradoja.

En un ambiente tan cambiante por su incertidumbre, complejidad y diversidad, algunas técnicas administrativas de organizaciones privadas pueden ser usadas para mejorar el desempeño de las instituciones públicas. Las propuestas de la liberalización comercial de los ochenta que sostenían que era buena para el crecimiento y con esto se reduciría la pobreza, son empíricamente cuestionables y de poco uso práctico para mejorar el diseño de la política y sus resultados en un mundo de altos niveles de complejidad e incertidumbre.

En el sector público, las reformas de organización y gestión estatal se orientan a lograr una mayor flexibilización de estructuras y procesos con la finalidad de resolver los problemas de contingencias que los altos niveles de incertidumbre y complejidad del medio ambiente representan, a involucrar una mayor participación social y a diferenciar los procesos de regulación y de gestión de los servicios públicos.

Las nuevas formas organizacionales tratan de responder a la interdependencia, los requerimientos de flexibilidad, la velocidad, el poder y el desmembramiento.

\section{a) Absorción de incertidumbre}

Lo que constituye la globalización es la interacción que cambia los escenarios para los individuos, las organizaciones y la sociedad, que se encuentran constantemente acosados por fuerzas contradictorias e incertidumbres. El campo organizacional incide en la construcción social de la realidad constituida por la objetivación de intercambios tipificados o categorización de las organizaciones, los cuales reducen la ambigüedad e incertidumbre.

En cualquier interacción humana se presenta la incertidumbre estratégica la cual puede ser constreñida a la conducta. La racionalidad de las reglas en condiciones de incertidumbre justifica el uso de guías de acción y reglas para determinar la conducta bajos ciertas situaciones. El neoinstitucionalismo histórico de North (1991a,b) combina las teorías del comportamiento humano con los análisis de los costos de transacción a partir de la consideración de que las instituciones son una creación humana para absorber incertidumbre de las interacciones en situaciones de alta complejidad.

Las organizaciones y las instituciones reducen los niveles de incertidumbre y mejoran la predictibilidad de la conducta humana mediante el establecimiento de las reglas del juego que estructuran las interacciones entre los humanos. Es decir, las instituciones son reglas de acción, expectativas y normas que determinan parcial o totalmente mediante incentivos y sanciones el comportamiento de los individuos en las organizaciones de una sociedad. Las instituciones y organizaciones políticas determinan el tipo de relaciones de la sociedad política y la dinámica de la sociedad civil, que pueden ser de incertidumbre.

Las organizaciones son un conjunto de reglas formales e informales que afectan el desempeño económico debido a que su razón de ser es la creación de orden y reducción de incertidumbre de los intercambios, reducen los costos de transacción e inducen a comportamientos cooperativos. Las organizaciones e instituciones tienen 
como funciones reducir la incertidumbre ambiental, resolver los conflictos, estabilizar y equilibrar los intereses de los agentes económicos y actores políticos, dar continuidad a los proyectos, inducir a las personas a organizar sus actividades, etc

Por tanto, la principal función de las organizaciones es reducir la incertidumbre a través de importantes elementos como asegurar la propiedad y los contratos de derechos. El desempeño económico es el resultado de una mezcla de organizaciones e instituciones formales e informales que juegan un papel importante en la determinación de los costos y que generan predictibilidad al limitar la incertidumbre de los comportamientos económicos. En sí, se requieren cambios en las metas de las organizaciones para responder a la incertidumbre, el enfoque estratégico en el diseño de procesos y estructuras, un énfasis en lo social e interpersonal y una reemergencia de la legitimidad

En condiciones de incertidumbre, las probabilidades no se atan a un número finito de eventos como en el caso de situaciones de riesgo donde es posible calcular los valores esperados bajo el marco de referencia de la teoría de las decisiones. En condiciones de incertidumbre, la racionalidad perfecta tiene poco sentido, por lo que Simon (1955) denominó como racionalidad limitada. En este sentido, Hermalin (1999) sostiene que la formalización de una cultura corporativa permanece como un fundamento para categorizar y entender posibles comportamientos futuros imprevisibles de contingencias ambientales hasta que se desarrollen modelos más aceptables que reduzcan la incertidumbre de las contingencias, y en términos más generales, modelos de racionalidad delimitada.

En las nuevas formas, la organización jerárquica da forma a la operación horizontal, procesos interfuncionales y empoderamiento de pequeñas unidades que tienden a autoorganizarse más rápido que las grandes unidades, dando forma a la organización caórdica que se refiere a su habilidad para generar orden del caos y para arribar a soluciones constructivas en ambientes de alta incertidumbre.

Por ejemplo, cualquier diseño organizacional de las políticas de capital humano debe enfatizar la heterogeneidad en habilidades, la incertidumbre de los retornos y la necesidad de tomar en cuenta tanto la heterogeneidad como la incertidumbre en los análisis de costo beneficio para el desarrollo de las habilidades. La heterogeneidad y la incertidumbre de los retornos son elementos perversos de las inversiones de capital humano, por lo que la reducción de la incertidumbre tiene beneficios sustanciales.

Un estudio organizacional de la política de capital humano centrado en los fundamentos económicos y científicos mejora en un acercamiento empírico las políticas de evaluación de los programas y políticas previamente experimentadas. Debido a que el capital humano es una buena inversión, es importante tomar en cuenta la dinámica del ciclo de vida de la adquisición del aprendizaje y habilidades en el diseño de políticas efectivas. De esta manera, un análisis comprensivo de las políticas de capital humano debe tomar en cuenta todo el conjunto de instituciones que contribuyen a su desarrollo, tales como, la familia, las escuelas y las organizaciones.

Otro buen ejemplo del impacto de la incertidumbre en las nuevas formas organizacionales es el incremento gradual de la demanda por la protección del ingreso y la provisión de servicios sociales mediante arreglos del Estado de Bienestar y de las limitaciones en los procesos de toma de decisiones políticas que deben ser secuenciales para los diferentes actores. Los intereses de los actores se ajustan al bien común mediante la búsqueda de intereses compartidos que combinan los intereses de los participantes y que los implica en una dependencia mutua.

El reconocimiento del carácter antagónico entre actores requiere de habilidades para el manejo de la incertidumbre, la complejidad y la diversidad. Las personas experimentan cada vez más diferencias culturales debido a la globalización y junto con la fragmentación crean retos de identidad, inseguridad, ansiedad, incertidumbre. Para enfrentar los retos que plantean la diversidad sociocultural es necesario desarrollar habilidades para la administración de esa diversidad para manejar la incertidumbre y la identidad.

La teoría de las organizaciones y el análisis de las políticas públicas caracterizadas por su nivel de incertidumbre, controversia y consenso fundamentan teóricamente el estudio de gobernanza mediante la 
diferenciación de estructuras organizacionales que legitiman su diseño. La gobernanza de las instituciones trata de los arreglos que gobiernan las relaciones de los individuos en las instituciones; la legitimación de formas de gobernanza se orienta a la absorción de los niveles de incertidumbre que facilitan la realización de consensos y reducen la controversia.

Los nuevos mecanismos de gobernanza que superan los sistemas de regulaciones existentes son el resultado del desarrollo de nuevas teorías económicas y de su instrumentación mediante el poder político de regímenes conservadores. Las nuevas formas institucionales surgen y se combinan con los arreglos institucionales para crear nuevas estructuras y procesos organizacionales.

El diagnóstico de los problemas organizacionales en los procesos de toma de decisiones también identifica las estructuras formales e informales de gobernanza, en donde tanto los fines y medios se caracterizan por los niveles de incertidumbre, consenso o certidumbre y controversia. El diseño organizacional de las estructuras formales e informales de decisiones mediante procesos de diferenciación, tanto al interior y entre las organizaciones mismas, se orienta a lograr un desempeño en función de una racionalidad en la coordinación y ejecución de las actividades y absorción de incertidumbre.

Las redes informales que desarrollan confianza, alivian la incertidumbre inherente a negociaciones con extraños, mientras que la existencia de instituciones legales formales que exigen el cumplimiento de los contratos representan menos riesgos para las negociaciones realizadas fuera de la estructura de las redes informales. Por otro lado, las redes cooperativas responden a la incertidumbre mediante la generación de mecanismos de reputación de los individuos que al interactuar con otros establecen estrategias de castigo mediante el supuesto de que consideran las acciones futuras, pero también establece límites al tamaño de los grupos informales, es decir, la cooperación se torna más difícil de sostener en grupos informales más grandes, aunque los beneficios sean mayores para los individuos por las posibilidades de encontrar más socios.

El aprovechamiento de las economías de escala a partir de innovaciones tecnológicas y especialización requiere del diseño de complejas estructuras de arreglos institucionales que faciliten las interrelaciones y negociaciones de los diferentes actores y absorban los niveles de incertidumbre

Las nuevas formas y arreglos institucionales requieren de características y capacidades que absorban las incertidumbres de las acciones corrosivas de los mercados.

\section{b) Complejidad de sistemas}

La creciente complejidad del entorno sectorial enfrentado por las organizaciones provoca estructuras internas crecientemente diferenciadas y complejas, tales como los sistemas de redes para compartir diferentes tipos de información, datos, conocimientos y activos, en donde el funcionamiento de cualquier parte de la estructura se entiende en función de las demás.

Si la cantidad de información relevante se incrementa, las decisiones incrementan en complejidad lo que demanda grandes esfuerzos cognitivos. Es esa complejidad lo que no sólo hace muy difícil la programación temporal de los cambios sino que producirá también casi inevitablemente cambios no intencionados y efectos imprevistos. La selección individual de las estrategias de elección puede balancear el deseo de lograr precisión con el deseo de minimizar los esfuerzos, lo que da lugar a que las decisiones se vuelvan más complejas mientras que los tomadores de decisiones adoptan estrategias más simples de elección para enfrentar la complejidad, a pesar de que el incremento de información causa un incremento en la selectividad de la información procesada.

Las fuentes obvias de complejidad causan que los decisores adopten estrategias de decisión simples de entre un número determinado de alternativas y un número determinado de atributos relevantes para cada alternativa, es decir, a pesar de un gran número de alternativas, los tomadores de decisiones emplean estrategias sencillas.

La economía de los costos de transacción se desarrolla de acuerdo con las consideraciones de las complejidades de la conducta humana y siempre trata de encontrar las aparentes explicaciones a las comparaciones de las 
estructuras institucionales. En particular, los costos y beneficios de los sistemas alternativos de colocación de recursos se ven impactados por la mayor complejidad y especialización de la actividad económica, la creciente interdependencia de muchos mercados de productos intermedios.

La descentralización tiende a la desaparición progresiva de las fronteras entre la complejidad e interdependencia de lo público y lo privado. La nueva gestión pública incluye los procesos de descentralización en todos los niveles de gobierno, aunque en su implementación no siempre se considera las complejidades locales.

Las nuevas formas de organización y relación entre el Estado y la sociedad civil se encuentran más bien sujetas a una multiplicidad de dinámicas complejas regidas por los intereses y fines del mercado. El análisis de las asimetrías entre la expansión y contracción del gasto en beneficios sociales bajo los arreglos del Estado de Bienestar lleva a fuertes discusiones debido a los procesos políticos que generan la irreversibilidad cuando se trata de limitar los beneficios sociales implantados. Por lo tanto cada nuevo tipo de beneficio que se implanta tiende a crear un nuevo grupo de intereses de beneficiarios que tiende a acentuar los procesos de irreversibilidad y dificulta la remoción de un arreglo de formas organizacionales sin que no haya complicaciones en otros arreglos.

La sociología política describe la complejidad y la fragilidad de la inserción de la sociedad civil en la esfera pública y endereza las criticas hacia el modelo de desarrollo neoliberal impuesto por el estado. Es el sistema social el que provee el principio de organización económica y las reglas de gobernabilidad para hacer funcionar la competencia del mercado como un mecanismo social. Sistemas transociales dan forma y penetran en los diferentes niveles en forma cambiante y compleja, afectando la estructuración del campo para influenciar y controlar la conducta de las organizaciones. Es así que la identidad colectiva tiende a reducir la complejidad, por ejemplo. De acuerdo a Melucci (1996:70) la identidad colectiva es un proceso por el que se "construye un sistema de acción. La identidad colectiva es una definición interactiva y compartida producida por un número de individuos (o grupos, en un nivel de mayor complejidad) respecto de las orientaciones de su acción y el campo de oportunidades y constreñimientos en el que tal acción tiene lugar."

\section{c) Flexibilidad de estructuras, comportamientos y procesos}

La economía estratégica pueden dar mayor flexibilidad a los países en desarrollo. La flexibilidad de una combinación dinámica de recursos tiene lugar dentro de las fronteras formales de las organizaciones y entre estas fronteras. Las nuevas formas de organización del trabajo basados en sistemas flexibles postfordistas modifican las relaciones entre los factores del capital y el trabajo para establecer nuevos equilibrios en las fuerzas sociales con base en el predominio de la acumulación capitalista apoyada en la innovación tecnológica. Las nuevas formas de organización postfordista neoliberales se fundamentan en los avances de la revolución técnico científica que transforman las barreras espaciales y temporales para acelerar los patrones de generación, reproducción y acumulación del capital.

El capital transnacional requiere de un nuevo diseño institucional y organizacional supranacional en un sistema económico y político global que facilite sus procesos de operación y acumulación flexible. El Estado transnacional crecientemente desarrolla mecanismos militares para la ejecución de la política económica, pero ha sido incapaz de regular los flujos de capitales especulativos que generan incertidumbre e inestabilidad al propio sistema capitalista.

El surgimiento de un modelo de desarrollo híbrido debe administrar en todos los niveles de gobierno, en forma flexible, la coordinación y armonización de los intereses públicos con los privados y los de la comunidad. Se necesitan incorporar constructos a nivel de comunidad para entender los efectos de los cambios en las formas organizacionales. La fortaleza de los actores sociales que dirijan y propicien procesos de cambio, facilita el involucramiento de las comunidades en el diseño organizacional e institucional e implantación de proyectos, en el seguimiento y cumplimiento de resultados mediante acciones de accountability. Ejemplos de modelos organizacionales que incorporan estas características son la organización postemprendedora y la firma flexible. 
El Estado como articulador de la gobernabilidad en las esferas económicas, sociales y políticas está en constantes transformaciones discontinuas conforme a un nuevo diseño neoliberal. El viejo modelo de gestión pública burocrática basada en una racionalidad normativa ha cedido paso a un modelo de gestión más flexible orientado por una racionalidad tecnocrática. Por lo tanto, el paradigma burocrático de la administración pública considerado como el modelo de la teoría organizacional que tenía como finalidad la normalización dela racionalidad instrumental para la eficiencia, tienen alcances limitados en aquellos países donde no existen las condiciones económicas, políticas, sociales y culturales, dando lugar a un modelo de administración que se centra en la racionalidad de una estructura organizacional que se adapte con mayor flexibilidad a los cambios y contingencias.

La racionalidad normativa muestra un movimiento de la organización a la institución, que legitiman las elecciones organizacionales en referencia a la misión y valores de la organización que sostiene la integridad organizacional. Sin embargo, en relación a la racionalidad normativa, las ventajas de las normas legales sobre las morales son la facilidad para establecerse, la flexibilidad y la magnitud de las sanciones. La falta de especificidad y flexibilidad de las reglas morales conduce a errores con más facilidad que las legales.

Las reformas de organización y gestión estatal se orientan a lograr una mayor flexibilización de estructuras y procesos con la finalidad de resolver los problemas de contingencias que los altos niveles de incertidumbre y complejidad del medio ambiente representan, a involucrar una mayor participación social y a diferenciar los procesos de regulación y de gestión de los servicios públicos. La reforma del Estado fortalece los gobiernos para realizar prácticas más efectivas de coordinación, comunicación, flexibilidad y participación, que tienden a la eficiencia adaptativa pública y privada, promueve el aprendizaje social para la formulación e implantación de políticas públicas que garantizan un mejor desempeño del crecimiento económico y desarrollo social.

Sin embargo, en un proyecto de nación se definen las áreas organizacionales de la provisión de bienes y servicios que deben ser atendidos por el Estado mismo y otras en las que la determinación de su carácter público o privado resulta ser más conflictiva. El gobierno, por lo tanto, es visto como un conjunto de organizaciones e instituciones que por medio de sus estructura burocráticas tiene como principal función la de prestar los servicios públicos, los cuales son demandados por la ciudadanía a través del proceso de votación. El mecanismo de votación mayoritaria determina las políticas públicas en un sistema democrático, aunque considerado como un proceso de elección colectiva, los resultados no son del todo consistentes con las elecciones sociales y tienden a incrementar el nivel de gasto del Estado.

Las instituciones y organizaciones pueden percibirse como soluciones de equilibrio a los problemas de elección colectiva. Quizás por esto, en el nuevo contexto, los gobiernos democráticos no conceden demasiada autonomía a los procesos de diseño e implementación de las políticas públicas. Con la descentralización organizacional e institucional, los municipios vuelven a asumir un rol protagónico en la administración de la provisión de los servicios públicos como atención a las necesidades de las localidades. Los procesos de descentralización se orientan a transferir recursos del sector público central a los gobiernos locales y de conformidad con sus características propias debe permitírseles administrarse sus recursos con mayor flexibilidad en sus mecanismos financieros.

Existen muchas confusiones todavía en el terreno de las relaciones interorganizacionales entre servicio público y ciudadano, para definir al usuario o consumidor de los servicios, cliente, público, etc. así como hasta que niveles de participación ciudadana en los procesos de planeación, diseño y entrega de los servicios públicos por los niveles de juzgamiento que implican. Además, el Estado debe diseñar las políticas públicas tendientes a eliminar las discriminaciones de acción de los diferentes agentes económicos y a regular las variables del medio ambiente organizacional e institucional.

La gestión pública busca la eficacia a nivel macro a través de los cambios estructurales en las relaciones multiorganizacionales. La gestión pública del Estado transforma sus estructuras, comportamientos y procesos burocráticos para ser sustituidas por arreglos institucionales más flexibles que facilitan la participación más directa de la comunidad y de la sociedad civil en los asuntos públicos mediante la promoción de 
comportamientos solidarios, procesos deliberativos democráticos y mecanismos de transparencia y rendición de cuentas.

La nueva gestión pública es un proceso de reingeniaría y rediseño de las relaciones entre los actores involucrados en la esfera estatal para la creación de valor público mediante el fortalecimiento de las instituciones a partir del aumento del capital social y el aprendizaje organizacional que promueven espacios de confianza entre el sector público, la ciudadanía y la sociedad civil. El papel fundamental de la sociedad civil es constituirse en los agentes del cambio social mediante la promoción de la democracia y una mayor participación en los procesos de toma de decisiones públicas. Como una fuerza, la sociedad civil puede hacer rendir cuentas a políticos y burócratas. No obstante Finot (1999) concluyó que "sólo excepcionalmente se consiguió la participación de los ciudadanos en la gestión pública, además de en las elecciones".

Los procesos flexibles de gestión pública necesitan ser democratizados mediante la consolidación de la participación de los ciudadanos en la búsqueda de alternativas para una correcta y eficaz toma de decisiones que garantice su aplicación. La participación de los ciudadanos en los procesos de formulación, iniciativa e implantación de las políticas públicas es limitada, ocasional y sujeta a procesos débilmente institucionalizados. Los procesos de la gestión pública requieren de un rediseño flexible para la creación de nuevas capacidades de las instituciones políticas orientadas a promover la participación y acción de la sociedad civil en los asuntos públicos locales mediante la formación de un capital social.

Una gestión pública de calidad alienta la sinergia social orientada a incrementar los servicios a la sociedad a través de profundos cambios en las estructuras institucionales y organizacionales del gobierno y del establecimiento de condicionantes como el establecimiento de una sociedad con un ambiente de legalidad, civilidad y cumplimiento de la ley, actualización constante de la normatividad de la administración pública para adecuarse a los cambios del entorno y los valores, de los movimientos sociales y de los nuevos procesos políticos, demandas sociales, tendencias en la participación y corresponsabilidad ciudadana, etc. Sin embargo, la calidad de la administración pública no siempre es garantía de la calidad de aplicación de la política económica.

La aplicación estratégica en el ámbito de la administración pública de técnicas de gestión de calidad para mejorar su propia actuación y para lograr un uso más eficiente de los recursos requiere del diseño flexible de estructuras organizacionales institucionales que acomoden las demandas de una ciudadanía más participativa y una sociedad más democrática en ambientes más inestables y turbulentos de los fenómenos de globalización económica.

El enfoque de calidad en los procesos, más que en el cliente o servicio, es el más aceptado y más conveniente. Las respuestas que proporcione la administración pública con soluciones efectivas y de calidad a las demandas sociales deben además estar caracterizadas por el interés público, la transparencia y honestidad, pero sobretodo alcanzar eficientemente los objetivos relacionados con la mejora del bienestar social, a pesar de las limitaciones impuestas por las condiciones macroambientales, las disfuncionalidades del mercado y la práctica de técnicas de gestión pública. Por ejemplo, la flexibilidad se incrementa cuando la cooperación se realiza mediante outsourcing y subcontratistas y facilitan el acceso a capacidades, experiencias y activos especializados.

El neoliberalismo es hábil para presentar las exigencias inflexibles de contratos de trabajo leoninos bajo la apariencia de la "flexibilidad". Los poderes políticos retroceden ante el avance del mercado, y se convierten en meros ejecutores de una política económica diseñada conforme a los requerimientos del libre mercado, desregulación, privatización, bajos impuestos a empresas y flujos de capital, flexibilidad laboral, etc. El programa de la globalización que el empresariado viene promoviendo desde los años setenta es regresivo, caracterizado por una reducción de la intervención pública, movilidad y flexibilidad de los trabajadores, ayuda pública a la inversión privada mediante asistencia fiscal, reducción de los aportes patronales, etcétera.

Con una orientación a la privatización directa y la remercantilización laboral para reforzar el papel del mercado en una sociedad postindustrial basada en la flexibilidad laboral que considera que los sistemas de protección social no son bienes colectivos. Bajo el supuesto del "secuenciamiento", para entrar a la reforma de la nueva 
gerencia que promueve una mayor flexibilidad en el empleo público, era necesario haber cubierto completamente la etapa de los servicios públicos que implicó el servicio civil en los administradores de carrera.

Para que los ciclos económicos sean sustentables, se requiere de regulaciones y seguros sociales anticíclicas, equilibrios fiscales y mejora del gasto público, regulaciones de los ingresos de capitales, flexibilidad cambiaria, el uso del encaje y fondos de estabilización, además de promoción de exportaciones. Un mercado de capitales desregulado y con flexibilidad cambiaria limita hasta cierto punto, la política económica de los Estados y les obliga a un juego de competencia y no cooperación modelado por el dilema del prisionero que da como resultado que limiten su soberanía nacional.

\section{d) Interdependencia e interacciones de agentes económicos y actores políticos y sociales}

La interdependencia organizacional es el grado en que una organización opera en forma interdependiente con otras organizaciones restringidas por los limites impuestos por las capacidades de procesamiento de la información y la distancia física. La interdependencia cuestiona los supuestos de las ventajas que representan el control de recursos dentro de las fronteras de una organización específica. El nivel de interdependencia que prevalece ahora no tiene precedentes.

Los resultados de cualquiera de las partes se relacionan con las acciones y los resultados de otros agentes, por lo que el cambio de un componente de un sistema interdependiente produce cambios impredecibles en otros. Existe una relación estrecha entre la institucionalización de las normas y el comportamiento individual y organizacional, es decir, las instituciones se legitiman cuando incentivan a los individuos en las organizaciones. Por ejemplo, el sistema de méritos es cuestionado como un sistema de incentivos efectivo para lograr la eficiencia y la responsabilidad en la función pública.

El uso de formas de organización modulares se fundamenta en esta interdependencia de organizaciones mediante el uso de alianzas, arreglos de trabajo alternativo, tales como las organizaciones contingentes y contratos de manufacturas. La imagen e identidad de los grupos son parte de los recursos críticos de las organizaciones modulares.

Todo esto sucede en los tiempos en que las alianzas estratégicas, las inversiones conjuntas, las fusiones y las adquisiciones adquieren relevancia como el rostro más visible de la globalización económica y como formas de organización y producción que ofrecen una urgente respuesta a la necesidad que tienen las empresas para crecer y ganar poder en los mercados globales.

En una nueva era de demanda homogeneizada de los mercados globales, las corporaciones requieren de habilidades para colocar en los mercados productos estandarizados de alta calidad y precios más bajos que los competidores mediante el uso de economías de escala en los procesos de producción, distribución, administración y mercadotecnia a pesar de que los patrones de consumo pueden diferir marcadamente entre las regiones y países. El aprovechamiento de las economías de escala a partir de innovaciones tecnológicas y especialización requiere del diseño de complejas estructuras de arreglos institucionales que faciliten las interrelaciones y negociaciones de los diferentes actores y absorban los niveles de incertidumbre.

El diseño óptimo de las organizaciones toma en consideración los costos / beneficios de las diferentes estructuras organizacionales basadas en una coordinación óptima de interacciones entre las diferentes actividades. Las formas organizacionales horizontales logran eficiencias de alcance y escala. Las teorías afirman las interacciones estratégicas como el fundamento para el equilibrio múltiple entre las organizaciones y las instituciones, cuyos resultados no adscriben valores de óptimo. El crecimiento se presenta en una interrelación inestable entre las economías de escala y la especialización con los costos de transacción. La economía de los costos de transacción proporciona las base para la economía mediante el diseño de estructuras de las nuevas formas de organización y por lo tanto, las consecuentes formas de gobernabilidad.

Bajo el principio de la ventaja comparativa el desarrollo es considerado como un juego de suma no cero donde todos los actores ganan del comercio. De acuerdo a Porter (1990, p.73) creador de la teoría de la ventaja 
competitiva, la prosperidad de las naciones se crea, no es el resultado de los recursos con que cuenta el país, su mano de obra, sus tasas de interés o su valores de cambio.

Veliyath y Zahara (2000), ofrecen el siguiente esquema para explicar las relaciones existentes entre globalización, cualidad emprendedora y competitividad.

\section{Globalización, cualidad emprendedora y competitividad}

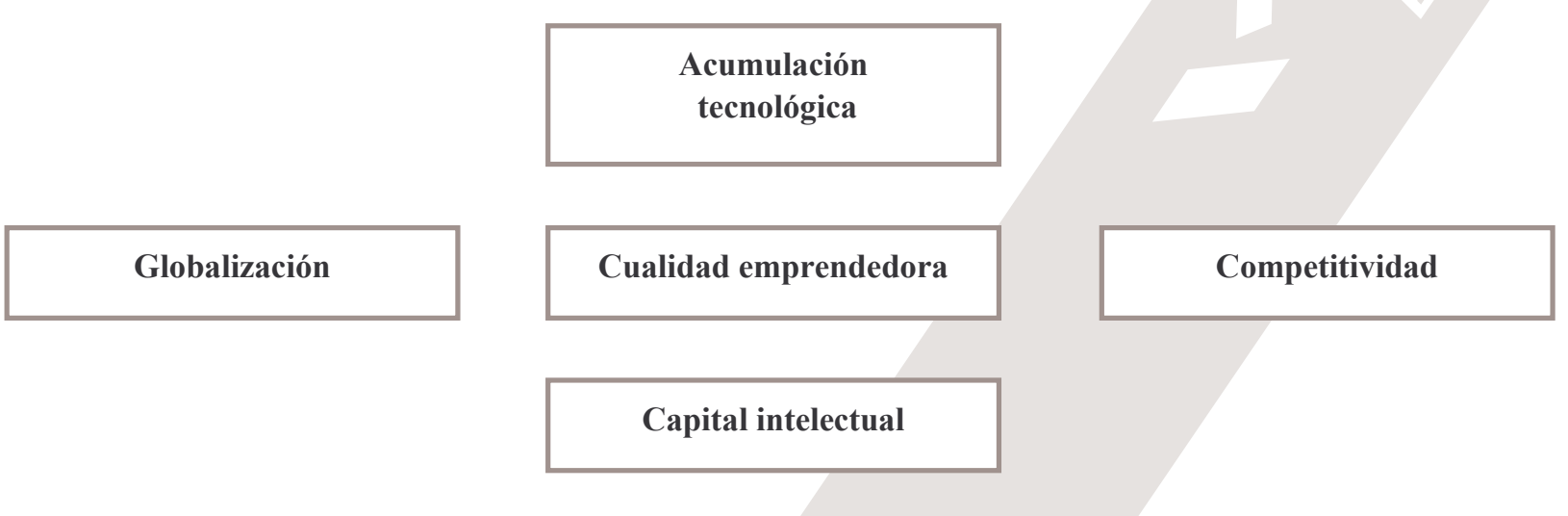

Los teóricos de este campo del conocimiento ofrecen varias alternativas de sistemas organizacionales en los cuales las tecnologías de la información representan un papel importante en la diseminación y aglutinamiento de tareas a través de la dispersión espacial y temporal. Las capacidades centrales de las organizaciones dependen del conocimiento tácito que contienen e inseparable de la tecnología de las organizaciones que constituyen la ventaja competitiva desde la perspectiva basada en los recursos que no son imitables o transferibles y que por lo tanto no pueden adquirirse en los mercados.

Este acercamiento se asocia con la teoría evolucionista de la firma. Las instituciones orgánicas son el resultado impredecible de la evolución gradual de la persecución de intereses individuales. Por lo tanto es el capital intelectual que las organizaciones adquieren, promueven, desarrollan, cultivan y aprovechan del poder cerebral de sus miembros y trabajadores, lo que constituye la base de su competitividad.

Como dimensiones de la competitividad se consideran entre otros factores el precio, la capacidad de innovación y desarrollo de nuevos productos y tecnologías y la calidad de las instituciones domésticas. Con respecto a la competitividad basada en el precio se determina en función de los costos locales en relación a los costos foráneos. La capacidad de innovación y desarrollo se relaciona con la calidad y la motivación del capital humano. Y la calidad de las organizaciones locales con el desarrollo de instituciones sociales y jurídicas que contribuyen al incremento de la productividad de la fuerza de trabajo, reducen los conflictos sociales, etc.

En los mercados globales, las interdependencias y las interacciones entre las empresas y los consumidores, culturas y capitalismos, transforman las preferencias hasta homogeneizarlas, lo cual provoca que la gente reaccione positiva o negativamente en las expresiones de fundamentalismos. Los microsucesos son la forma de acción e interacción subjetiva en la que tanto por rutina y presencia sistémica toma forma la estructura social que institucionalizan los sujetos en la interacción (Giddens 1984:170-173). Para otros la divergencia y la heterogeneidad es una forma válida de reacción frente a la mercadización de la vida social y la integración comercial y financiera. Las estrategias de desarrollo empresarial tienden a orientar la producción hacia los sectores que tienen ventajas competitivas y un mayor valor agregado para insertar eficazmente la economía local y regional en los flujos de los mercados internacionales.

\section{e) Incremento de velocidad}

Una mayor velocidad es la característica de todos los aspectos de las funciones de las organizaciones, desde las comunicaciones internas al desarrollo de productos para el intercambio competitivo. La velocidad tiene efectos 
en el decrecimiento de las imperfecciones del mercado, el incremento de la volatilidad a que deben responder las organizaciones y el decremento de los tiempos de estímulo respuesta involucrados en actividades organizacionales prosaicas.

\section{f) Cambios del poder}

Los cambios en el poder derivan de la posesión de activos tangibles e insumos de poder derivados de la posesión del conocimiento e información. Las nuevas asimetrías del poder son un reto clave para las nuevas formas organizacionales. Los acuerdos de la economía global no pueden ser tomados solamente entre un grupo de burócratas y negociadores comerciales lejos de los intereses de las mayorías de la población. La transferencia del poder del capital del Estado-nación al espacio global requiere el diseño de instituciones supranacionales para escapar del control de las instituciones locales y nacionales.

Los grupos de interés buscan mantener y acrecentar su poder en un determinado espacio de las estructuras institucionales y organizacionales mediante su posicionamiento como actores estratégicos en un determinado sistema sociopolítico. La capacidad de demanda de algunos grupos de interés sobre el Estado limita el pretendido universalismo del bien público, para adoptar estrategias basadas en tendencias focalistas, contingentes, descentralizadas y de terciarización del sector privado y la sociedad civil.

Así, una gran parte de la población mundial queda excluida del acceso a una de las más importantes fuerzas impulsoras de la globalización. Sin embargo, la influencia no es unidireccional. El conocimiento moviliza, fortalece y emancipa a la sociedad civil otorgándole autonomía para el ejercicio de sus funciones orientadas a urgir tanto a las organizaciones del Estado como a las privadas que incluyen las grandes corporaciones transnacionales, para que cumplan con sus responsabilidades y posibilita el empoderamiento (empowerment) de las comunidades para encontrar soluciones a sus propios problemas.

Por lo tanto, los procesos de formulación de las políticas públicas son el resultado de la relación del ejercicio y comportamiento del poder entre los diferentes actores y grupos de interés. Cruz (2002) argumenta que "la justificación del poder es un ejercicio central en la legitimación de la acción gubernamental, y su realización (y existencia) constituye para los actores implicados el punto central de debate sobre la política y sobre las políticas públicas que los gobiernos diseñan y ponen en marcha."

Por ejemplo, la socialdemocracia impulsa el Estado de Bienestar que perpetúa la injusta distribución del poder, no necesariamente se identifica con la libertad y la justicia social y cuyas relaciones sociales derivan de la explotación del hombre por el hombre a través de los procesos productivos que limitan el desarrollo de las potencialidades de los individuos. La política de bienestar socialdemócrata centra la responsabilidad en las organizaciones e instituciones del Estado para desmercantilizar la provisión de los beneficios sociales sobre la base de principios de universalidad, igualdad y cobertura global.

Este modelo de bienestar es incompatible con el apremiante avance del capitalismo desregulado, ante cuyas presiones de adaptación, la orientación del bienestar social de la socialdemocracia activamente han redireccionado la aplicación de recursos y servicios a las familias jóvenes. Este modelo genera una menor desigualdad y eleva el nivel de vida a través de formas organizacionales orientadas a la procuración de mejores servicios públicos, lo que finalmente repercute en aumento de la productividad y satisfacción laboral. Sí el gasto en protección social universal bajo el modelo socialdemócrata no necesariamente implica una reducción en la competitividad, antes por el contrario, la eleva. La crítica del libertarismo al Estado de Bienestar se endereza contra las estructuras burocráticas centralizadas que aniquilan la participación ciudadana en el diseño de los beneficios como reconocimiento a la satisfacción de las necesidades individuales

Poder y rendición de cuentas para su uso fueron puestos por los propietarios para su control por los altos directivos de las organizaciones burocráticas tradicionales, se ha convertido en un asunto más complejo en aquellas organizaciones donde existe una multiplicidad de accionistas y cuya forma de organización es más horizontal. 
La escuela de la economía de los derechos de propiedad argumentan que la propiedad común de los bienes genera ineficiencias, pero sin embargo, se puede contra argumentar que la privatización también es ineficiente ya que otorga más poder para negociar a aquellos que adquieren la propiedad privada. Estos propietarios se convierten en dominantes porque obtiene mayores beneficios del disfrute de bienes, sobretodo cuando estos son de carácter público. El enfoque neoinstitucionalista de la economía provee un marco de referencia teórico que enriquece a los derechos de propiedad, que apoyan el diseño de estructuras de gobernabilidad de acuerdo a la combinación de distintos costos de transacción y competencias, problemas de agencia y contratos internos y que además toman en cuenta el medio ambiente institucional.

Cuando el medio ambiente institucional no proporciona el marco de referencia para que las transacciones se realicen adecuadamente, con costos bajos o para proteger y garantizar los derechos de propiedad de los diferentes peligros, entonces se requiere del diseño de estructuras y mecanismos de gobernabilidad. La desmoralización es la erosión de una forma particular de definir la moral, asociado a papeles que resultan de cambios en el medio ambiente institucional.

En primer término hay que considerar las diferencias que presenta el mismo sistema presidencialista en diferentes realidades si consideramos las atribuciones y poderes constitucionales del presidente, su relación y control con los partidos políticos y el apoyo que recibe, los poderes legislativos, los poderes reactivos y proactivos para modificar el status quo. El sistema de partidos no ha podido sustituir las funcionales del presidencialismo como árbitro para la resolución de conflictos y disputas que surgen entre los diferentes actores con intereses económicos, sociales y políticos.

El debate gira en torno a si existe una relación de causalidad entre el régimen y la democracia y si las características y factores del diseño organizacional e institucional del régimen parlamentario funcionan mejor que las democracias presidencialistas, las cuales se concentran en su mayor parte en Latinoamérica donde las diferencias socioeconómicas y políticas son muy marcadas. De acuerdo con Levy and Spiller (1996, pp. 4-6), las características del medio ambiente incluyen la existencia de un sistema judicial independiente, respetado y no corrupto, así como de equilibrios y contrapesos al poder ejecutivo que incluyen la Constitución, las cámaras legislativas, y los niveles múltiples de restricciones gubernamentales e internacionales.

El derecho es un instrumento del Estado que constituye un sistema de incentivos y obstáculos que encausan los comportamientos en determinados cursos de acción de conformidad con los objetivos que un sistema económico y social se plantea para su proceso de desarrollo. Por lo tanto, el derecho establece los límites propios al poder estatal en el ámbito económico y su capacidad de intervención, posibilitan el diseño de los arreglos institucionales que facilitan la predictibilidad en las interacciones sociales e incentivan los comportamientos que concurren al modelo de desarrollo deseado. A partir de los fundamentos teóricometodológicos de las relaciones entre el desarrollo económico y las instituciones jurídicas que establece la Nueva Economía Institucional, se superan las limitaciones del análisis del "movimiento del derecho y el desarrollo", y se establecen las bases para la construcción del Estado de Derecho promovente del desarrollo económico.

\section{g) Desmembramiento de fenómenos organizacionales}

Los procesos de globalización aunado al crecimiento incontrolable de megalópolis en algunos países menos desarrollados crean nuevas formas de organización y desorganización que someten a la población a una brutal competencia de tal forma que establecen similitudes y diferencias en donde se mezclan rasgos de la modernidad y la posmodernidad marcadas con la realidad de las sociedades desarrolladas. Las manifestaciones multiculturales en estas sociedades hasta cierto punto configuran estos rasgos que por un lado desintegran la identidad individual y las referencias comunitarias, destruyen las estructuras familiares y sociales, así como las manifestaciones religiosas, culturales e intelectuales. Estas reacciones consideradas como irracionales frente a los excesos racionalistas de la organización, se encuentran estrechamente vinculada con el ambiente económico, social y político. 
Existe un vacío entre el desarrollo rápido de nuevas formas organizacionales en práctica y la capacidad de las perspectivas existentes en la teoría. Los conceptos de organización postburocrática, postmoderna, la organización postemprendedora y la firma flexible se refieren a nuevos principios organizacionales y expresan los nuevos paradigmas en las formas organizacionales. Otros aspectos específicos de estos paradigmas incluyen el federalismo, la corporación virtual, la corporación reingenierada, la compañía creadora de conocimiento, la organización "ambidexterus", de alto desempeño o sistemas de trabajo de alto compromiso, la organización híbrida y la "solución transnacional", etc.

La coherencia y la motivación son proveídas por una visión y una cultura. Así también, el creciente significado de activos creados, como por ejemplo, las habilidades humanas y las capacidades tecnológicas en los procesos de valor agregado, el desarrollo de nuevas instituciones y formas organizacionales, y una reevaluación de culturas y normas conductuales, han impactado los costos y beneficios de los sistemas alternativos de colocación de recursos. La estructura social de las sociedades contemporáneas experimentó cambios profundos, entre los cuales el más importante es la disminución, en la industria, de los obreros frente a los "operadores", quienes, más ricos en capital cultural, podrán concebir nuevas formas de organización, nuevas armas de lucha y nuevas solidaridades.

\section{Diseño organizacional e institucional}

El diseño organizacional de las estructuras formales e informales de decisiones mediante procesos de diferenciación, tanto al interior y entre las organizaciones mismas, se orienta a lograr un desempeño en función de una racionalidad en la coordinación y ejecución de las actividades y absorción de incertidumbre. El sistema de medición del desempeño estratégico es uno de los componentes de la Nueva Administración Pública.

El diseño y operación de las organizaciones e instituciones se fundamenta en el hecho de que proporcionan los incentivos económicos que los individuos requieren para guiar su comportamiento, de tal forma que les permita maximizar sus beneficios mediante la mayor reducción de los costos de transacción. Por lo tanto en el diseño organizacional e institucional deben considerarse aquellas transacciones en donde los involucrados reciban beneficios mutuos.

El diseño organizacional institucional de un país es determinante para su desarrollo económico, social y político y condicionan las consecuencias del sistema político. El nuevo diseño organizacional e institucional se caracteriza por nuevos valores y estructuras mentales, nuevas reglas y tipos de relaciones entre los diferentes agentes y actores. El diseño organizacional e institucional eficaz incentiva las conductas que maximizan los resultados y que repercute en el desempeño económico. De acuerdo a Weaver y Rockman (1993) las respuestas que los países recientemente democratizados tengan ante los retos y desafíos que les plantea el desarrollo económico y la integración política y social de sus pueblos, dependerán en medida importante de la opción de diseño institucional que adopten.

Soltan (1993, p. 4) advierte que “...cuando hablamos de una 'perspectiva del diseñador' institucional, puede sonar como el lenguaje de los ingenieros sociales, dándole forma a las instituciones del modo que otros ingenieros diseñan puentes y barcos o del modo que los arquitectos diseñan edificios. El problema del diseño institucional es claramente muy distinto, así que adoptar una perspectiva de diseñador no necesariamente debe conducir al gran constructivismo que Popper, Hayek y muchos otros han acertadamente criticado, y cuyos peligros son obvios".

Las instituciones políticas determinan los procesos de formulación e implantación de decisiones y políticas públicas las que a su vez determinan en forma contingente las capacidades de los gobiernos. Para Weaver y Rockman (1993), la capacidad de los gobiernos es el "patrón de influencia gubernamental sobre los contextos que produce sustancialmente resultados similares a través del tiempo y en áreas de políticas". El PNUD la define como la "habilidad de individuos y organizaciones o unidades organizacionales para rendir funciones efectiva, eficiente y sosteniblemente. La eficiencia gubernamental medida a través de la habilidad de los gobiernos queda potencialmente condicionada por el diseño institucional que el país escoja " (Lasagna, 2001). 


\section{a) Enfoque neoliberal del diseño organizacional}

En el enfoque neoliberal, se intenta construir un orden social desde los procesos de la economía global. El diseño organizacional de las instituciones pragmáticas se hace en función de contratos deliberados que tienen como base la teoría de la información imperfecta y los costos de transacción. La información estratégica que tienen los diferentes agentes económicos y actores explica su comportamiento estratégico en la racionalidad del diseño organizacional de los arreglos institucionales. El estudio de la racionalidad facilita el análisis de la estabilidad de los arreglos organizacionales, su inercia, cambio y conflicto.

La eficiencia resulta de alinear los costos de transacción con las estructuras de gobernabilidad. Las estructuras de gobernabilidad organizacional se relacionan con los atributos de las transacciones, ya que los mercados se desplazan en forma selectiva más que uniformemente. En el diseño de las estructuras organizacionales con base en los costos de transacción deben considerarse aquellas transacciones que proporcionen beneficios mutuos a las partes involucradas. Las transacciones tienen diferentes necesidades de adaptación autónoma o cooperativa.

Los principios en que se sustenta el diseño del Estado mínimo definen la caracterización de sus estructuras burocráticas formalistas, procesos de toma de decisiones públicas altamente jerarquizados y centralizados, obsesión por la búsqueda de una racionalidad operativa que homologa los trámites administrativos y despersonaliza o trata por igual a los ciudadanos, la búsqueda afanosa de la calidad de los procesos que encarecen la entrega de servicios y bienes públicos sin que concurran los correspondientes beneficios, etc. Los procesos caóticos de privatización se justifican con el diseño de un Estado pequeño y eficiente, sin que necesariamente implique funcionar de una forma diferente, ya que ignora el fundamento de a quien sirve. Los grandes agentes económicos y actores pueden iniciar acción y subsidiar otros participantes.

Las preferencias de los agentes económicos y actores políticos son cruciales para lo que se diseña organizacional e institucionalmente porque los participantes conocen que los altos costos pueden involucrarse si las decisiones van en contra de ellas. Las ilusiones acerca de los bajos costos de cooperación pueden prevalecer. Un asociacionismo denso en un medio ambiente político y económico que propicie el respeto de los valores e intereses de todos los agentes económicos y actores sociales y políticos, son las condiciones básicas de todo diseño organizacional e institucional sistema liberal democrático.

El nivel organizacional e institucional, social o de medio ambiente se enfoca en los procesos de legitimación y significado. De acuerdo a Easton (1992), los procesos de legitimación requieren de apoyo difuso que proporciona la legitimidad de las instituciones del sistema político y de apoyo específico manifiesto por determinada acción política. Cuando los agentes económicos y los actores políticos tienen mayor acceso a información pueden desarrollar mayor capacidad para argumentar y justificar sobre la base de las instituciones, los procesos de toma de decisiones públicas para el diseño y aplicación de las políticas públicas, logrando con ello un nivel más elevado de legitimidad.

El desarrollo político o gobernanza ocurre en todas las instancias del sistema político y orienta los cambios con fundamentos en la democracia liberal. La participación de los agentes económicos y los actores en las situaciones políticas locales crean las condiciones necesarias para el diseño de los arreglos institucionales que faciliten la gobernabilidad. La gobernanza se entiende como la capacidad del gobierno para guiar, dirigir e influir en las acciones económicas y sociales. Prats (2001) delimita las dimensiones de gobernanza en la estructural que hace referencia a "los arreglos institucionales existentes en una sociedad dada" y en la dimensión dinámica o de proceso que se refiere a "las acciones de los actores" que afectan la estructura. La dimensión normativa de gobernanza delimita el papel que deben desempeñar los diferentes actores, y más específicamente las instituciones, en la promoción del desarrollo. Este concepto normativo considera a la gobernabilidad como "una condición necesaria y no suficiente para la producción de desarrollo".

El desarrollo centrado en los paradigmas del crecimiento económico no es sustentable y por lo tanto es necesario tomar en consideración la relación del crecimiento con equidad, de tal forma que se preserven los recursos naturales para las futuras generaciones mediante la generación de las condiciones de beneficio de todos del ciclo de crecimiento. El impacto de las crisis financieras en la pobreza, la desigualdad y el desarrollo 
sustentable ha aumentado en la misma proporción en que las crisis se han profundizado y se han hecho más frecuentes. El desarrollo es la condición de satisfacción de las necesidades de la población de una nación mediante el uso racional y sustentable de los recursos naturales.

Los espacios vacíos que dejan los arreglos institucionales son fuente recurrente de conflictos que desestructuran los sistemas sociopolíticos. El argumento convencional contra el acercamiento es que los diseños organizacionales e institucionales no están realmente diseñados en el sentido que hay una relación directa entre las intenciones de los actores políticos y los resultados institucionales, pero son más o menos el resultado de la evolución histórica (Kaiser, 1997).

Mediante un nuevo diseño institucional sustentado en cambios constitucionales, se amplia la distribución del poder a las organizaciones sociales y privadas. La linealidad de las reformas en los procesos de desarrollo, así concebidas, también son cuestionadas con la necesidad de reformar las reformas.

\section{b) Enfoque intergubernamental del diseño organizacional}

Para los proponentes del intergubernamentalismo, el diseño institucional de las nuevas formas y estructuras de gobernabilidad del orden económico global se concentra en la fundación, consolidación y participación de organismos multilaterales creados y controlados por los Estados nación como los actores principales, sin considerar la participación de los actores privados. Las capacidades enfocan la atención en la importancia de reconciliar las tareas organizacionales e institucionales a nivel nacional y el diseño organizacional de instituciones de gobernabilidad internacional en forma que produzcan efectos de suma positiva.

La regulación intergubernamental adquiere la forma de una globalización macropolítica cuando los Estados nacionales delegan competencias y soberanía a los organismos multilaterales en los que operan una estructura de gobernabilidad semijerárquica y una forma basada en la negociación intergubernamental. La importancia que para los intergubernamentalistas adquieren la gobernanza local y regional como base de las normas globales y la competitividad en los procesos de integración económica, se fundamenta en las interacciones locales del mercado, el Estado y los actores sociales.

El Estado crea las arenas en las cuales promueve las relaciones entre los agentes económico locales e internacionales y, además, hace cumplir las reglas del juego. En el escenario local se optimiza la participación individual, pero la acción colectiva resulta disfuncional. La acción colectiva reduce la racionalidad económica debido a malos diseños de organizaciones, inadecuados sistemas de incentivos económicos y elevación de los costos de transacción.

El reto más grande que enfrentan las instituciones de gobernabilidad internacional es revertir las tendencias de desigualdad creciente entre los diferentes países y hacia el interior de los mismos, que caracterizan a los procesos actuales de globalización económica. La simple apertura económica difícilmente logrará estos resultados. Si todas las instituciones internacionales trabajan hacia las mismas metas, se reducirá la dificultad para la resolución de conflictos que se presentan en los acuerdos comerciales.

\section{c) Diseño organizacional de redes de gestión}

Como alternativa a la controversia entre los neoliberales que procuran más mercado y los intergubernamentalistas que buscan una mayor participación de estado, surgen las estructuras de redes de las sociedades que involucran en su diseño las estructuras de los mercados, los actores privados (organizaciones no gubernamentales, empresas, clusters, redes de gestión política, etc.) y los Estados en nuevas formas de gobernabilidad, tales como las interacciones entre los emplazamientos locales, nacionales y globales, las alianzas trans-locales. Por lo tanto, en los contextos de las formas y estructuras de gobernabilidad global de los mercados que interacciona con la gobernabilidad local, se integran en procesos de globalización económica los emplazamientos y clusters locales y regionales

En las redes de gestión política global interaccionan actores privados y públicos que forman estructuras horizontales de cadenas globales de valor agregado y constelaciones transnacionales con estructuras 
multiniveles, que como formas de gobernanza influyen en los flujos transfronterizos de inversión, tecnología, producción, distribución y consumo. De acuerdo a Castells, el Estado-red plurinacional es el Estado real actual que surge de la red de los Estados-nación y que requiere de una nueva legitimidad política. La estructuración flexible del Estado-red en el concepto de Castells (1998) se combinan los principios de subsidiariedad, flexibilidad, coordinación, participación ciudadana, transparencia administrativa, modernización tecnológica, transformación de los agentes y retroalimentación en la gestión.

Las cadenas globales de valor agregado tienen como soporte una compleja diversidad de estructuras de gobernabilidad que van más allá de la simples transacciones comerciales, tal como Humphrey y Schmitz (2001:7) argumentan: "Las estructuras de gobernabilidad encadenadas constituyen las relaciones y los mecanismos por medio de los cuales se consigue la coordinación de la cadena más allá del mercado".

La gobernanza es un producto conformado por redes intra e interorganizacionales que implica los fenómenos de la co-dirección y la co-gestión bajo mecanismos de responsabilidad, transparencia y rendición de cuentas instrumentadas mediante el diseño de estructuras de gobernanza inclusivas y supervisadas por el gobierno.

La organización virtual descansa en los pilares de la producción de información, la estandarización de la interacción y la abstracción de los derechos de propiedad (Mowshowitz, 1994). La esencia de la organización virtual es la administración de una actividad orientada por metas de forma que es independiente de los medios de su realización. Al igual que en las organizaciones virtuales, en las organizaciones del denominado Estado contractual, Estado sombra, o Estado virtual los contratos sustituyen a las estructuras jerárquicas, dando lugar a una diversidad de arreglos organizacionales.

\section{Los retos}

Las formas de organización alternativas difieren en sus procesos de transformación intrínsecos e intertemporales y requieren de un cuerpo metodológico conceptual de economía organizacional para su buen diseño. El prestigio de la organización es importante para impulsar los cambios en cualquier campo organizacional.

Los procesos de globalización dan lugar a la creación de redes del mercado integrada por las relaciones de las elites capitalistas transnacionales que se movilizan desde arriba y a redes de contrapoderes y resistencia transnacional que se moviliza desde dentro y desde abajo, mediante mecanismos de alianzas y asociaciones, para contrarrestar los alcances de la otra. La formación de alianzas entre las personas basadas en la solidaridad e identidad a través de redes de conocimiento permitirá una mayor movilización política de las comunidades para ejercer acción colectiva contra prácticas nocivas tanto del gobierno como de las grandes corporaciones transnacionales. La protesta en los tiempos actuales se caracteriza por una madeja de organizaciones coordinadas en nuevas formas de organización en red que enfatizan los códigos culturales y los roles de identidad de las comunidades.

Las comunidades que comparten valores éticos son las formas organizacionales más efectivas, por lo que las personas que las forman pueden ser las más eficaces sin que renuncie a su comunidad. Las comunidades epistémicas se forman con miembros que comparten las mismas posiciones ideológicas, uniforma los contenidos mediante la exclusión de las confrontaciones que limitan la calidad de las decisiones y motiva la formación de las advocacy coalitions o coaliciones de promoción.

En las mismas formas de organización social que están estrechamente vinculadas con la política, ocurre la estructuración de la sociedad civil que fomenta relaciones asociacionales animadas por ciertos valores ideales democráticos para dar sustento a la participación ciudadana. Un asociacionismo que coordine los intereses entre la comunidad y los sectores privado y público en los tres niveles de gobierno, supera la dicotomía que considera al Estado propietario como opuesto a los intereses del mercado identificado con el sector privado. Una administración pública orientada por servir a lo público, al colectivo como origen de su desempeño obliga a repensar y a rediseñar sus funciones y procesos bajo nuevas formas de organización que propicien la transparencia y rendición de cuentas y tome en consideración las opiniones de los ciudadanos y los involucre en 
los procesos de toma de decisiones y formulación e implantación de las políticas públicas. Los procesos de formulación de las decisiones y políticas públicas están estrechamente relacionados con las organizaciones y son el resultado de los procesos institucionales.

Los ideólogos ortodoxos neoliberales limitaron las funciones de regulación de actividades, de protección y bienestar del Estado. Así, la función principal del nuevo Estado regulador es mantener la estabilidad de los mercados financieros para proteger los movimientos especulativos de los capitales de la volatilidad de los mercados emergentes, mediante la movilización de recursos financieros de otras fuentes, pero principalmente los provenientes de mayores cargas impositivas a contribuyentes con ingresos bajos. Cómo debe involucrarse el Estado en el desempeño de estas actividades y mediante qué métodos y formas de organización para hacerlo eficazmente, es una de las consideraciones del debate actual.

Los nuevos movimientos sociales se desarrollan como redes más que como organizaciones estables en períodos activos y en períodos latentes de actividad modificando sus características y presencia mediante la construcción de identidades colectivas. Como un proceso dinámico de construcción y negociación de los elementos de la identidad colectiva que implica definiciones cognitivas respecto de las finalidades, los medios y el campo de acción, se refiere a una red de relaciones activas entre actores que interactúan, se comunican, negocian o toman decisiones, $\mathrm{y}$, finalmente, requiere una cierta inversión emocional que permita a éstos sentirse parte de una unidad común (Rodríguez Girat, 2002).

La propuesta está dada con la creación de un movimiento internacional que diseñe nuevas formas de organización social para la producción y consumo, así como para la formulación de políticas alternativas a las neoliberales hegemónicas. El posneoliberalismo puede orientarse a la satisfacción de necesidades sociales mediante procesos de desmercantilización ya sea dentro de la misma lógica o en contradicción del sistema capitalista

Otros aspectos a considerar son el acelerado movimiento hacia una economía orientada por la información y la innovación, y la ampliación de las fronteras territoriales de las empresas. Las organizaciones legítimas y competitivas tienden a innovar a partir de un estándar y por tanto contribuyen a legitimar los cambios de los adoptadores de las innovaciones.

La solución transnacional es una visión de una red integrada en la cual el centro corporativo guía los procesos de coordinación y cooperación entre las unidades subsidiarias en un clima de toma de decisiones compartidas, mezcla la jerarquía con la red y retiene la creación del valor en una corporación (Bartlett and Ghoshal, 1998). La organización como una red de mentes distribuidas, en donde la autoridad, el poder, la responsabilidad y los recursos son descentralizados en equipos de unidades semiautónomas que consisten de staff semiautónomo, profesional y conocedor. 


\section{Referencias}

Antrás, Pol y Helpman, Elhanan. Global sourcing, Manuscript, 2003.

Bartlett, G.A. \&t Ghoshal, S. Mamaging acrros borders: The transnacional solution (2nd edition), Boston: Harvard Business School Press, 1998.

Coase, R.H. The nature of the firm (First published in 1937). In O.E. Williamson \& S. G.Winter (Eds.), The nature of the firm: origins, evolution, and development: 18-33. New York:Oxford University Press, 1991.

Castells, Manuel. ¿Hacia el estado red?. Globalización económica e instituciones políticas en la era de la información", en Seminário Internacional sobre Sociedade e Reforma do Estado. Brasilia: Mare, 1998.

Cruz, César Nicandro. "La justificación del poder en el proceso de las políticas públicas: implicaciones de la gobernabilidad democrática", Desarrollo Humano e Institucional en América Latina, Edición No. 27, feb. 2002.

Easton, D. "Categorias para el análisis sistémico de la política", en W.A.A. Diez textos básicos de ciencia política. Barcelona. Ariel. Ciencia Política, 1992.

Finot, Ivan (1999) "Elementos para una reorientación de las políticas de descentralización y participación en América Latina". Revista del CLAD Reforma y Democracia, No. 15, Oct. 1999.

Giddens, A. The constitution of society. Los Angeles. University of California Press, 1984.

Hermalin, E. Benjamin. "Economics \&t corporate culture", Draft. Cornell University and University of California, Berkeley, 1999.

Humphrey, J. y Schmitz, H. 'The Triple C Approach to Local Industrial Policy', World Development, Vol 24, 12, pp.1859-1877, 1996.

Kaiser, André. "Types of democracy. From classical to new institutionalism", Journal of Theorethical Politics 9(4): 419-444, 1997.

Lasagna, Alberto. Comentario. Reseña a "Do Institutions matter?, Government capabilities in the United States and abroad". Biblioteca de Ideas. Instituto Internacional de Gobernabilidad, 2001. http://www.iigov/org/iigov/pnud/bibliote/resenas/resena0004.htm

Levy, B. and Spiller, P. eds. Regulations, institutions and commitment: Comparative studies of telecommunications. New York: Cambridge University Press, 1996.

Melucci, A. Challenging codes Collective action in the information age. Cambridge:Cambridge University, 1996.

Mowshowitz, A. Virtual organization: A vision of management in the information age. Information Society, 10: 267-294, 1994.

North, D. Transaction cost, institutions and economic history. Zeitschrift für die gesamte Staatswissenschaft 140, pp. 7-17, 1984.

Selznick, P. Leadership in administration: A sociological interpretation, New York: Harper and Row. 1957.

Porter, Michael. The Competitive Advantage of Nations. Harvard Business Review. March-April. Boston, 1990.

Prats, Joan. "Gobernabilidad democrática para el desarrollo humano. Marco conceptual y analítico", Instituciones y Desarrollo, No. 10, Oct. 2001. Barcelona, España, 2001. http://www.iigov.org/revista/re10/re10_04.htm.

Rodríguez Giralt, Israel. El efecto de las TIC en la organización de la acción colectiva: la virtualización de los movimientos sociales, 2002.

Selznick, P. TVA and the grass roots. Berkeley: University of California Press, 1949.

Simon, Herbert A. "A behavioral model of rational choice," Quarterly Journal of Economics, 69: 99-118, feb. 1955.

Soltan K. "What Is the New Constitutionalism?", en Elkin S. y Soltan K., eds., 1993. A New Constitutionalism, The University of Chicago Press, Chicago, 1993.

Veliyath, Rajaram and Zahara Shaker A. "Competitiveness in the 21st century: Reflections on the growing debate about globalization", Advances in Compettitiveness Research, Vo. 8, No. 1: 14-33, 2000. 
Weaver R. Kent y Rockman, A. (eds.) "Do institutions matter?. Government capabilities in the United States and Abroad. The Brookings Institution, Washington, DC, 1993.

Williamson, Oliver E. "Why law, economics, and organization?", Business and Public Policy Working Paper BPP-81. Institute of Management, Innovation and Organization, University of California, Berkeley, 2000.

Williamson, O.E. "Economic institutions: Spontaneous and intentional gobernanza," Journal of Law, Economics and Organization, 7, 1991a.

Williamson, O.E. Strategizing, economizing, and economic organization, Strategic Management Journal. $1991 \mathrm{~b}$. 